Kapanidou, M, Curtis, N and Bolanos-Garcia, V

Cdc20: At the Crossroads between Chromosome Segregation and Mitotic Exit.

Cdc20: At the Crossroads between Chromosome Segregation and Mitotic Exit. Trends in Biochemical Sciences, 42 (3). pp. 193205.

doi: 10.1016/j.tibs.2016.12.001

This version is available: https://radar.brookes.ac.uk/radar/items/76f31141-6b51-40de-a058-35894db214bd/1/

Deposited on RADAR: March 2017

Copyright (C) and Moral Rights are retained by the author(s) and/ or other copyright owners. A copy can be downloaded for personal non-commercial research or study, without prior permission or charge. This item cannot be reproduced or quoted extensively from without first obtaining permission in writing from the copyright holder(s). The content must not be changed in any way or sold commercially in any format or medium without the formal permission of the copyright holders.

This document is the postprint version of the journal article. Some differences between the published version and this version may remain and you are advised to consult the published version if you wish to cite from it. 


\title{
Cdc20: At the Crossroads between Chromosome Segregation and Mitotic Exit
}

\author{
Maria Kapanidou ${ }^{1}$, Natalie L. Curtis ${ }^{1}$, and Victor M. Bolanos-Garcia ${ }^{1}{ }^{*}$
}

${ }^{1}$ Faculty of Health and Life Sciences, Department of Biological and Medical Sciences, Oxford Brookes University, Oxford OX3 OBP, UK *Correspondence: vbolanos-garcia@brookes.ac.uk (V.M. Bolanos-Garcia).

Cell-division cycle protein $\mathbf{2 0}$ homologue (Cdc20) has important functions in chromosome segregation and mitotic exit. Cdc20 is the target of the spindle assembly checkpoint (SAC) and a key cofactor of the anaphase-promoting complex or cyclosome (APC/C) E3 ubiquitin ligase, thus regulating APC/C ubiquitin activity on specific substrates for their subsequent degradation by the proteasome. Here we discuss the roles of Cdc20 in SAC signalling and mitotic exit, describe how the integration of traditional approaches with emerging technologies has revealed new details of Cdc20 functions, comment about the potential of $\mathrm{Cdc2O}$ as a therapeutic target for the treatment of human malignancies, and discuss recent advances and controversies in the mechanistic understanding of the control of chromosome segregation during cell division.

\section{Cdc20 Roles in Mitosis}

The SAC (see Glossary) is an intricate signalling system that prolongs mitosis until all chromosomes achieve correct bipolar attachments to spindle microtubules. A proper SAC response involves communication with the kinetochore, a multiprotein structure that constitutes the site for attachment of chromosomes to the microtubule polymers that pull sister chromatids apart in dividing cells $[1,2]$. Defects in chromosome segregation can lead to aneuploidy, a prevalent characteristic of human cancer cells, and genome instability [3-5]. Here we describe the importance of Cdc20 in the SAC, the overall structural organisation of $\mathrm{Cdc20}$, how multiple protein motifs in this protein act in a concerted fashion to ensure the regulation of mitosis progression and mitotic exit, the physiological implications of abnormal Cdc20 expression in humans, and the potential of this APC/C regulator as a drug target for innovative anticancer therapies. In humans the SAC is orchestrated by the serine-threonine kinases budding uninhibited by benzimidazoles 1 (Bub1), Bub-related1 (BubR1), monopolar spindle1 (Mps1), and Aurora B and the proteins mitotic arrest deficient-like 1 (Mad1), mitotic arrest deficient-like 2 (Mad2), Bubrelated 3(Bub3), andBub3-interacting GLEBS-motif-containing ZNF207 (BuGZ). Cell-division cycle protein 20 homologue ( $C$ dc20) is a modulator of the ubiquitin E3 ligase APC/C (see Figure IA in Box 1) the cellular activity that drives mitotic exit and as discussed below, a bona fide component of the SAC. The SAC inhibits this activity in response to chromosome misorientation and/or misattachment to spindle microtubules. SAC proteins are recruited to the kinetochore, inducing conformational changes in Mad2 that promote its binding to Cdc20 and BubR1 (known as Mad3 in yeast and worms) to form the mitotic checkpoint complex (MCC), a potent inhibitor of the APC/C (see Figure IB in Box 1) [6]. Activation of the $A P C / C$ requires the binding of its cofactor $C d c 20$. Once all sister kinetochores are properly attached and bioriented, the SAC is satisfied and the chromosomes congress to the metaphase plate, which in turn leads to disassembly of the MCC and the release of $A P C / C$ inhibition. In the latter process, the $C d c 20-A P C / C$ interaction is greatly enhanced by phosphorylation of core protein subunits of the APC/C by cyclin-dependent kinases ( $\mathrm{dks}$ ). Phosphorylated APC/C can activate and recruit its substrates cyclin $B$ and securin for polyubiquitination and their eventual destruction by the proteasome. Molecular recognition of cyclin $B$ and securin by the $A P C / C$ is mediated by a short protein motif termed the destruction box (D-box) comprising the consensus sequence RxxLxxxxN [7]. Degradation of cyclin B results in cyclin B-Cdk1 inactivation and the subsequent onset of cytokinesis [8-10]. Therefore, $\mathrm{Cdc} 20$ is a protein with a pivotal role in the regulation of chromosome segregation and mitotic exit. As discussed below, the dual, antagonistic functions of Cdc20 in the SAC and as an APC/C cofactor place this protein at the crossroads of key signalling events that control cell division in eukaryotic organisms. 


\section{Multiple Motif Organisation of Cdc20}

Specific motifs found throughout the Cdc20 polypeptide chain are responsible for the interaction of this protein with SAC central components and APC/C subunits during the critical transition from metaphase to anaphase. Cdh1, the second activator and regulator of substrate specificity of the APC/C [11], shares a similar domain organisation with Cdc20 (see Figure IC in Box 1). Cdh1 can bind to securin during the G1 stage of the cell cycle, where the Cdc20 protein levels are low. Despite sharing similar domain organisations, the ways in which Cdc20 and Cdh1 interact with the same substrate differ [12]. This elicits the question: how exactly do the multiple motifs of Cdc20 dictate its specific yet versatile functions in mitosis? A good starting point is to describe $\mathrm{Cdc} 20$ as a protein that is organised in two main segments: an N-terminal region of low structural complexity and a C-terminal region that defines the WD40 fold. The $\mathrm{N}$-terminal disordered region contains C-box, KEN-box, and CRY-box motifs whereas a C-terminal tail after the WD40 domain contains the Ile-Arg (IR) motif (Figure 1). The KEN-box and the CRY-box act as two degradation signals (degrons) that function independently from each other. The KEN- and the D-box are key APC/C recognition binding sites [13-15]. The KEN-box motif (KENXXXN/D) is defined by the amino acid residues lysine (K), glutamate (E), and asparagine (N) (residues 26-28 in human Cdc20). Both the KEN- and the CRY-box motif function as APC/C- Cdh1-dependent degrons at the end of mitosis. The Cdc20 CRY-box includes the residue S170, which is phosphorylated by polo-like kinase-1 (Plk1), a protein also implicated in the regulation of diverse kinetochore and centrosome proteins. Phosphorylation of S170 by Plk1 is critical for the timely ubiquitination and destruction of Cdc20 [16]. Despite the fact the KEN- and CRY-box motifs are regulated by APC/C-Cdh1, at least in embryos and oocytes the Cdc20 degradation rate mediated by recognition of the CRY-box motif is greater than that involving the KEN-box motif [16]. The crystal structure of a truncated form of Cdc20 lacking the flexible $\mathrm{N}$-terminal region (PDB 4GGC) revealed a segment organised as a canonical WD40-repeat fold. This protein repeat plays critical roles in Cdc20 and Cdh1 through the physical interaction of the WD40 repeat with APC/C substrates $[12,17-20]$. Each WD40 repeat comprises four $\beta$ strands that define a seven-bladed b-propeller architecture [21]. Each blade is a $\beta$ sheet of four antiparallel strands, with strand order $\beta A, \beta B, \beta C, \beta D$, arranged around a central axis with pseudo sevenfold rotational symmetry (Figure $2 A$ ). The $\beta D$ strand of one blade intersects to define the strands $\beta A$ to $\beta C$ of the next blade. The $\beta D$ strand from the first repeat is the outside strand for the last blade, thus creating a closed structure. In a canonical WD motif, the Trp of this signature establishes a pattern of hydrogen bonds in a triad involving residues Ser/Thr, His, and Asp [22]. However, the canonical WD signature sequence is largely absent in human Cdc20, with blades one to seven terminating in the amino acid pairs WS, WD, HD, WP, WN, WK, and WR, respectively. The sequence divergence affects core contacts between blades. Cdc20 proteins from other species also deviate at key residues of the WD40 canonical signature. In human Cdc20 the only conserved element of the prototypic triad is the Asp/Asn-GIn sequence at the tip of one of the loops. The typical Trp, Ser, and His signature residues are only found in blade six and are conserved in all Cdc20 homologues (the exception being Cdc20 from zebrafish, in which these residues are substituted by Phe, Ala, and Asn, respectively). The top surface and the bottom face of the WD40 domain contain large loops. The blades surround a central channel that narrows marginally near the top face. In human Cdc20 the width from the top to the bottom face is ca $27 \AA$; the diameter is ca $48 \AA$. The crystal structure of Cdc20 in complex with a BubR1 KEN-box motif-mimic peptide (PDB 4GGD) provided the first atomic insights on the mode of interaction of $\mathrm{Cdc} 20$ with this multidomain SAC pseudokinase and protein component of the MCC. The crystallographic structure showed that in Cdc20 the KEN-box- and D-box-binding regions are mapped onto the top face of the seven-bladed b propeller in which the KEN-box motif adopts an irregular $3_{10}$-helix architecture. The interaction involves amino acid residues of the loop region that link the first and seventh blades of the Cdc20 propeller (residues 183-188 in human Cdc20), which also establish multiple contacts with neighbouring amino acid residues to render a shallow top surface. The Cdc20 183-188 region includes a triad defined by residues D184Y185Y186 that configures a KEN-box motif-binding site. The triad residues physically interact with the side chains of the KEN-box motif residues E27 and N28 of BubR1 giving place to an autoinhibited conformation, thus providing molecular details of Cdc20 molecular recognition and regulation by BubR1.

The C-terminal region of Cdc20 contains a conserved IR motif that is essential for Cdc20 recruitment to the APC/C [20, 23, 24]. Various mechanistic models of the role of the Cdc20 IR motif in the SAC have been proposed. One model postulates that $\mathrm{Cdc} 20$ binds to the APC/C Apc8/Cdc23 subunit where the SAC is unsatisfied, leaving the TRP motif of the APC/C Apc3 subunit available for the Cdc20-independent recognition of substrates such as Nek2A [13, 25]. When the SAC is satisfied, the IR motifs of $\operatorname{Cdc} 20$ and Apc10 bind to one of the two subunits of the Apc3 homodimer thus establishing a stable interaction with the APC/C [13, 26-28]. Interestingly, a truncated version of the APC/C regulator Cdh1 lacking the IR tail can still bind weakly to the APC/C through the C-box motif located in Cdh1 N-terminal region [24] suggesting that other Cdh1 regions are implicated in the productive interaction of Cdh1 with APC/C. 
Multiple phosphorylation sites exist in the Cdc20 N-terminal region, a feature that resembles APC/C regulation by concerted phosphorylation and dephosphorylation events [29]. Substitution of R132 for alanine impairs Cdc20 binding to human Mad2 [30] whereas mutation of residues S41, S42, S72, S92, S153, T157, and S161 for alanine result in reduced checkpoint arrest in mitosis, presumably due to the loss of Bub1-mediated phosphorylation [31]. Similarly, suppression of Bub1 protein production or expression of a Bub1 dead kinase both abolish Cdc20 phosphorylation and impair the SAC [31], confirming the importance of Cdc20 phosphorylation in the SAC. Furthermore, in addition to the aforementioned mechanisms of chromosome segregation regulation, an effective SAC response implicates a Cdc20-Bub1-Plk1 axis, as concluded from studies conducted in human cells expressing truncated and phosphomimicking Cdc20 mutants [32]. These studies showed that the non-catalytic domain of Bub1 can bind to Cdc20 and Plk1 and provides a structural framework for the phosphorylation of $\mathrm{Cdc} 20$ by the latter kinase [32]. This process, which seems to be required for the in vitro inhibition of APC/C-Cdc20 but appeared dispensable for the assembly of the MCC, defined an additional layer of regulation of the SAC.

A further interaction that is essential to elicit a proper SAC response is that of Cdc20 with BubR1. The interaction involves two Cdc20-binding regions in the latter protein: one N-terminal KEN-box motif and a short region defined by residues 490560 in human BubR1. Different authors refer to this second Cdc20-binding site as the C20BD region [33], the ABBA motif (an acronym that alludes to the motif in cyclin A, Bub1, BubR1, and Acm1 proteins) [34], or the Phe box (from the two phenylalanine residues that define it; FSIFDE in human BubR1) [35]. The latter report also described a putative C-terminal D-box that is important for BubR1 binding to Cdc20 [35]. Similarly, the interaction of Cdc20 with Mad2 is critical for the SAC. Mad2 is a 23.5-kDa protein that contains a Hop1p, Rev7p, and MAD2 (HORMA) domain of multiple conformational states, including an open (o-Mad2) and a closed (c-Mad2) conformation [36,37]. In the latter conformational state, Mad2 binds to Cdc20 and to its upstream regulator Mad1. The Cdc20-Mad2 interaction involves a Cdc20-binding region known as the Mad2-interacting motif (MIM) [38, 39]. The interaction induces a seatbelt-like structure in C-terminal Mad2 [38, 39]. The o-Mad2 conformation results in steric hindrance of the ligand-binding site caused by its C-terminal region, thus impeding the adoption of a seatbelt topology and, consequently, the binding of Mad2 to Mad1 or Cdc20 [38]. When the SAC is unsatisfied, the Mad1-Mad2 heterocomplex is recruited to unattached kinetochores, which in turn promotes the conformational transition of Mad2 from the open to the close state. The association of c-Mad2-Cdc20 with BubR1 and Bub3 forms the MCC (Figure 2B) [40]. Comparison of the structure of Cdc20 alone with that of the Cdc20 implicated in the MCC from fission yeast (PDB 4AEZ) revealed little conformational change on Cdc20 association with Mad2 and Mad3 (the yeast orthologue of BubR1). The only significant difference is a disordered-to-ordered transition of the Cdc20 MIM motif on ligand binding [41]. The mode of interaction between human Cdc20 and a surrogate peptide of BubR1

KEN-box motif was very similar to that observed between Cdc20 and Mad3 in the fission yeast MCC [41], indicating a mode of molecular recognition of the BUB kinases by $\mathrm{Cdc} 20$ that is evolutionarily conserved. Taken together, these structural features reveal $\mathrm{Cdc} 20$ as a small, multisubstrate protein that acts as an organiser centre in the regulation of the cell cycle and APC/C activity. In addition, the requirement for Cdc20 by the Bub1-Plk1 interaction, the conformational transition of Mad2, and the interaction with Mad2 and BubR1 to assemble the MCC, confirm Cdc20 as a bona fide component of the SAC. Multiple studies show that the subcellular localisation and functions of Cdc20 are tightly regulated through allosteric mechanisms implicating protein-protein interactions, concerted phosphorylation and dephosphorylation cascades, and other post-translational modifications including ubiquitination, thus situating $\mathrm{Cdc} 20$ at the crossroads of signalling events that ultimately ensure genome stability. The implications of $\mathrm{Cdc} 20$ as a cell signalling regulatory node that ensures the unidirectional flow of information during cell division are discussed below.

\section{Cdc20 and the Regulation of the APC/C}

The MCC exists both in a free state and stably bound to the APC/C [29, 42, 43]. The MCC binds and inhibits Cdc20-bound APC/C (APC/C-Cdc20), forming a large complex called APC/C-Cdc20-MCC that contains two copies of Cdc20. More than 50 sites are phosphorylated in the APC/C complex during mitosis [44-46]. Under such conditions, phosphorylation of APC/C by the Cdk1 kinase controls $\mathrm{Cdc} 20$ loading onto APC/C to form an APC/C-Cdc20 complex [42]. Further control of APC/C-Cdc20 activity in response to the status of the mitotic spindle involves fine-tuned cycles of $\mathrm{Cdc} 20$ synthesis and degradation with the dynamic association of the MCC and APC/C-Cdc20-MCC assemblies [1, 47, 48]. Two independent breakthrough studies reporting high-resolution (i.e., 6.1-4.2- $\AA$ resolution) cryoelectron microscopy (cryo-EM) images of APC/C-MCC complexes have revealed new clues about $A P C / C$ regulation with an unprecedented level of detail $[49,50]$. The studies showed that the MCC interacts with an APC/C region located in close proximity to the region where a $\mathrm{Cdc} 20$ subunit was previously 
docked (Figure 2C). The multidomain protein BubR1 acts as a pseudosubstrate inhibitor and extends around the two Cdc20 subunits in such a way that it occupies all degron-binding sites on both copies. In this manner recruitment of the MCC to APC/C-Cdc20 physically impedes the recruitment of APC/C substrates (see Figure IB in Box 1) resulting in the allosteric regulation of $A P C / C$ ubiquitin $E 3$ ligase activity. A large conformational transition leads to an active, open state in which the Cdc20 ubiquitination sites Lys485 and Lys490 become accessible to the priming E2 subunit UBE2C of APC/C complex thus contributing to the regulation of APC/C-Cdc20-MCC functions. Although the presence of Bub3, a protein involved in termination of the SAC [51,52], was confirmed in a biochemically reconstituted Cdc20-MCC assembly it could not be located in the electrondensity maps, suggesting that Bub3 associates with APC/C-Cdc20-MCC in such a way that it remains largely flexible on binding the assembly. Ultimately, the conformational dynamics of the interactions confer distinctive ubiquitination activities on the APC/C that enable exquisite regulation of mitosis. The overall similarity of EM maps of APC/C-Cdc20-MCC complexes with and without Bub3 raises the following questions. Is the association of SAC proteins to form the MCC and other subcomplexes sufficient to promote UBE2C-dependent ubiquitination of Cdc20? How exactly are the APC/C-Cdc20-MCC open and closed conformations switched in the cell? How exactly does Bub3 associate with APC/CCdc20-MCC to regulate SAC signalling? These are key aspects of cell division regulation that remain to be fully understood. Phosphorylation of Cdc20 impairs the ability of the APC/C to activate the chain-forming E2 subunit Ube2S [53]. The inhibitory phosphorylation on Cdc20 is released by PP2A-B56, a phosphatase that is recruited to kinetochores by BubR1. In addition, depletion of Knl1, the kinetochore anchor of BubR1, prevents PP2A-B56 and UBE2S binding to Cdc20. These findings suggest that $\mathrm{Cdc} 20$ coordinates the formation of a kinetochore-bound complex that contains BubR1, UBE2S, and PP2A-B56 in a phosphoregulated process. In fission yeast PP1 activity is required to reactivate PP2A-B55 and PP2A-B56, indicating that at least in this exit [54]. The picture emerging from the studies described above is one of finely controlled assembly and disassembly of APC/C-Cdc20 and the MCC that in turn enables allosteric regulation of APC/C activity. This is achieved through multiple, cooperative interactions that act in a concerted fashion to ensure tight regulation of chromosome segregation and cell cycle progression. In this manner the interactions that control APC/C ubiquitin E3 ligase activity can turn the catalytic subunit into a molecular switch that, depending on the precise requirements by the cell at that point in time, functions as an inhibitor or a substrate of APC/C-Cdc20. The evolutionary advantage that this intricate mode of regulation confers on eukaryotic organisms is evidenced by the severe adverse consequences of APC/C deregulation on genome stability, an aspect that is discussed below.

\section{Cdc20 in Disease}

Abnormal Cdc20 expression appears in a majority of human cancers, including oral squamous cell carcinoma [55], human bladder carcinoma [56], and pancreatic [57], colorectal [58], breast [59], brain [60], and lung cell cancer [61], supporting an oncogenic role for $\mathrm{Cdc20}$ in promoting tumorigenesis. Suppression of Cdc20 expression in lung cancer and hepatocellular carcinoma decreased cell proliferation and induced G2/M cell cycle arrest, and in the latter case positively correlates with TNM stage [61, 62]. Due to its association with clinical stage, Cdc20 has been proposed as prognostic biomarker in human non-small cell lung cancer (NSCLC) and colorectal cancer [61]. Cdc20 overexpression and poor cell differentiation also occur in human pancreatic cancer cells [63]. Depletion of Cdc20 enhanced cell cytotoxicity following treatment with paclitaxel and increased the sensitisation of pancreatic cancer cells to gamma irradiation [63], suggesting that Cdc20 could be a useful marker to monitor cancer progression and a potential therapeutic target for the treatment of this devastating disease.

A growing number of viruses have been shown to target the APC/C, including human cytomegalovirus (HCMV), human papillomavirus (HPV) E2, Orf virus (ORFV), and human T cell lymphotropic virus type 1 (HTLV-1) Tax [64]. The molecular strategies deployed by these viruses to infect human cells vary widely. Nevertheless, the manipulation of the APC/C by the viruses has the common feature of allowing them to sustain their replication [64]. Although the use of viral particles as novel tools to study the APC/C is in its infancy, they may be instrumental in enhancing our understanding of cell division in human cells and exploring the possibility of separating the functions of Cdc20 in the assembly of the MCC, regulation of APC/C activity, and the exit from mitosis. Towards this aim, it would be of great significance to clarify exactly how viral factors affect the activity of the APC/C and to identify all of the APC/C subunits and APC/C-Cdc20 regulators that they target. It would also be relevant to assess the global effect of the viruses on cellular metabolism in normal cells and tissuespecific tumours and define whether APC/C manipulation by viral factors can sensitise cancer cells to drugs and/or gamma irradiation and proton beam therapy. It would be equally important to assess the potential benefits of using viral factors for cancer therapy and establish their potential use as novel drug-delivery systems targeting the SAC-kinetochoremicrotubule axis more effectively. 
Earlier observations showed that the weakening of SAC core proteins inhibited tumour cell growth, suggesting the SAC signalling pathway as a promising pool of new anticancer targets (reviewed in [65]). Such findings stimulated the search for and development of inhibitors that target the catalytic site of the SAC kinases Aurora B and Mps1, some of which have entered clinical trials. Examples are: the Aurora kinase inhibitor AT9283, which is prescribed for the treatment of nonHodgkin's lymphoma (Phase I completed; NCT00443976); the inhibitor PF-03814735, which is used for the treatment of histologically or cytologically confirmed malignancies (Phase I completed; NCT00424632); and the Mps1 inhibitor BAY1161909 (in Phase I trial for the treatment of solid tumours; NCT02138812) [66, 67]. Unfortunately, the use of smallsized inhibitors that act as ATP-binding competitors, including those that target SAC kinases, has been of limited success. The lack of specificity of these molecules for the intended kinase target, which may result in significant side effects and rapid development of drug resistance in treated cells, remain major concerns [68]. Alternative approaches include microtubule inhibitors such as vinca alkaloids and taxanes, which have been used to treat breast, ovarian, and lung cancer [69]. However, the clinical efficacy of these compounds is limited because of the variable response to microtubule inhibitors by the treated cells $[70,71]$. The fact that Cdc20 knockdown can lead to SAC-independent mitotic arrest that is comparatively more effective in killing cancer cells together with the emerging description of APC/C regulation to a unprecedented level of detail should open up new opportunities for exploration of the therapeutic potential of Cdc20 as a drug target affecting mitotic exit in cancer cells. In principle, the targeting of specific Cdc20 protein-protein interfaces (PPIs) may be a better anticancer therapeutic strategy than perturbing the spindle assembly with spindle-perturbing drugs or the use of ATP analogues that bind to kinase domains. This is because the structural and physicochemical features of PPIs are far more diverse than those defining the catalytic and/or substrate-binding sites. Ideally, the disruption of a specific PPI must have little (if any) effect on other specific interactions mediated by the target protein, thus decreasing the risk of undesired side effects. For instance, targeting the Cdc20-BubR1 and/or Cdc20-Mad2 interfaces may allow finetuning of the regulation of free and MCC bound Cdc20 protein levels, which may be effective in harnessing slippage in response to mitotic arrest or death in mitotic cells [72].

An excellent review discussing the potential of $\mathrm{Cdc} 20$ inhibition for the treatment of human malignancies listed 12 bona fide protein substrates of APC/C-Cdc20 and 13 substrates of APC/C bound to either the $\mathrm{Cdc} 20$ or the Cdh1 cofactor [73]. The wide scope of substrate molecules regulated by APC/C-Cdc20 confirms the prominence of APC/C as a master regulator of the eukaryotic cell cycle and the opportunity this represents for the treatment of human malignancies. Several smallsized compounds belonging to various chemical families (Figure 3) have been reported to inhibit APC/C functions. Their mode of action is summarised in Table 1 and discussed below. TAME is a mimetic drug of the IR motif involved in Cdc20 recruitment to the APC/C. TAME functions as a Cdc20 (and Cdh1) competitor of the Cdc20-APC/C interaction leading to inhibition of degradation of APC/C substrates [74]. Because TAME is not cell permeable, a prodrug called proTAME, which can be processed by in vivo by esterases to yield TAME inside the cell, has been developed. proTAME functions as an APC/C-Cdc2O inhibitor to retard tumour cell growth and induces mitotic arrest in the absence of spindle damage in a SACdependent manner. NAHA is a hydroxamic acid derivative that downregulates Cdc20 expression in breast cancer cells causing inhibition of cell proliferation and colony formation and reducing tumour weight in vivo [75]. However, the underlying molecular mechanism of action of this compound remains largely obscure [76]. GDMT is an alcohol found in a certain class of mushrooms that suppresses $\mathrm{Cdc} 20$ expression, hence inhibiting the growth and proliferation of aggressive breast tumours [77]. Apcin is a small-sized compound that prevents substrate recognition by Cdc20 leading to inhibition of ubiquitination of Cdc20 substrates [74]. Apcin binds to the pocket defining the D-box, thus blocking substrate-induced Cdc20 loading onto the APC/C [74]. Furthermore, apcin and a few apcin synthetic derivatives synergise with proTAME to inhibit mitotic exit in human colorectal and osteosarcoma cancer cells [74, 78]. Withaferin A stimulates Cdc20 and Mad2 degradation thus interfering with SAC function and extending mitosis. Ganodermanontriol (GDNT) downregulates Cdc20 expression and acts as an inhibitor of cell proliferation in breast cancer cells [77]. Moreover, a medicinal blend from mushrooms, Myco-Phyto ${ }^{\oplus}$ Complex, reportedly acts as a suppressor of the expression of cell cycle regulatory genes including Cdc20, thus inhibiting cell proliferation and invasiveness in breast tumours $[79,80]$. Regulation of Cdc20 expression by genistein seems possible in breast cancer cells [81]. CFM-4 is a compound that inhibits cell growth and invasion in malignant pleural mesothelioma [82]. In breast cancer cells, CFM-4-induced apoptosis may contribute to downregulation of $\mathrm{Cdc} 20$ [83]. More recently, the compound 6-brominated coumarin hydrazide-hydrazone derivative (BCHHD)-7c was reported to act as an inhibitor of Cdc20 expression in pancreatic cancer cells that were resistant to chemotherapy, suggesting that BCHHD-7c could be an effective antitumour drug for the treatment of pharmacologically induced drug-resistant cancer cells [84]. However, the precise effect of BCHHD-7c in cells overexpressing Cdc20 and the assessment of its potential therapeutic benefits for the treatment of cancer require additional in-depth investigations. 
None of the naturally occurring inhibitors reported to date (withaferin, GDNT, and genistein) are genuinely specific to Cdc20, so further studies are required to develop the molecules into truly specific Cdc20 inhibitors. By contrast, the synthetic compound apcin appears to be a specific Cdc20 inhibitor with a direct effect on APC/C-Cdc20 function. Due to the fact the inhibition of APC/C activation by apcin and TAME implicate distinct mechanisms, the combinatorial use of apcin and TAME can lead to a synergistic effect in human cancer cell lines [74]. Hence, the disruption of specific PPIs by simultaneously combining two or more drugs represents a promising new strategy for the treatment of cancer. Recent in vitro studies in Cdc20 knockdowns reported inhibition of the growth of human pancreatic carcinoma cells after the delivery of liposome-encapsulated Cdc20 siRNA [85]. These results suggest that siRNA methods can be an effective therapeutic strategy for the inhibition of tumour growth [85]. It can be anticipated that there will be a surge in the use of siRNA-based strategies targeting APC/C subunits, cofactors, and regulators - alone and in combination - together with the development of more effective methods for siRNA delivery in the coming years. The study of Cdc20 functions in the control of cell division has largely been focused on its interaction with the MCC and APC/C [50, 86-88]. By contrast, far less is known about the regulation of $\mathrm{Cdc} 20$ expression by upstream regulators, including p53 (which acts as a negative regulator, [89]); FOXM1 (a transcriptional activator), and possibly Emi1 and Usp44. It is encouraging that suppression of FOXM1 expression by shRNA decreased Cdc20 levels in glioblastoma, a prevalent and deadly type of brain tumour. While the APC/C cofactor Cdh1 has for some time been considered a suitable therapeutic target for the treatment of tumours caused by chromosome segregation defects, the potential therapeutic value of targeting Cdc20 (alone or in combination with other therapies) for the prevention of premature mitotic exit remains largely unexplored [65, 90].

\section{Concluding Remarks}

The use of emerging technologies such as single-molecule methods and super-resolution microscopy in combination with traditional biochemical, cellular, and structural biology approaches has revealed new details of the remarkable roles of $\mathrm{Cdc} 20$ in APC/C regulation in health and disease. These advances have provided new molecular details of the role of Cdc20 in the dynamics of SAC complex assembly and revealed Cdc20 to be a central organising centre for the accurate control of chromosome segregation and the timely end of mitosis. Future work should aim to unveil the functions of the different Cdc20 pools in the cell, provide a quantitative description of how changes in the concentration of the Cdc20 pools influence SAC signalling and mitotic exit in normal and tumour cells, and assess the therapeutic potential of targeting Cdc20 for the treatment of the diverse human cancers associated with chromosome segregation defects (see Outstanding Questions).

\section{Acknowledgments}

M.K. is supported by a Professor Nigel Groome PhD Studentship, Oxford Brookes University. This work was supported by Cancer Research UK (CRUK) grant number C5255/A18085 through the CRUK Oxford Centre. The authors thank Brenda A. Schulman for sharing cryo-EM maps and structure models of APC/C-MCC complexes before their publication. They apologise to those colleagues whose important contributions to this field could not be cited due to space limitations.

Supplemental Information

Supplemental information associated with this article can be found online at http://dx.doi.org/10.1016/j.tibs.2016.12.001.

\section{References}

1. Weir, J.R. et al. (2016) Insights from biochemical reconstitution into the architecture of human kinetochores. Nature 537, 249-253

2. Smith, C.A. et al. (2016) Human kinetochores are swivel joints that mediate microtubule attachments. Elife 5, e16159

3. Derive, N. et al. (2015) Bub3-BubR1-dependent sequestration of Cdc20Fizzy at DNA breaks facilitates the correct segregation of broken chromosomes. J. Cell Biol. 211, 517-532

4. Musacchio, A. (2015) the molecular biology of spindle assembly checkpoint signaling dynamics. Curr. Biol. 25, R1002R1018 
5. Sacristan, C. and Kops, G.J. (2015) Joined at the hip: kinetochores, microtubules, and spindle assembly checkpoint signaling. Trends Cell Biol. 25, 21-28

6. Lischetti, T. and Nilsson, J. (2015) Regulation of mitotic progression by the spindle assembly checkpoint. Mol. Cell. Oncol. 2, e970484

7. Glotzer, M. et al. (1991) Cyclin is degraded by the ubiquitin pathway. Nature 349, 132-138

8. Sivakumar, S. and Gorbsky, G.J. (2015) Spatiotemporal regulation of the anaphase-promoting complex in mitosis. Nat. Rev. Mol. Cell Biol. 16, 82-94

9. Braunstein, I. et al. (2007) Inhibitory factors associated with anaphase-promoting complex/cyclosome in mitotic checkpoint. Proc. Natl Acad. Sci. U.S.A. 104, 4870-4875

10. Burton, J.L. and Solomon, M.J. (2007) Mad3p, a pseudosubstrate inhibitor of APCCdc20 in the spindle assembly checkpoint. Genes Dev. 21, 655-667

11. Meghini, F. et al. (2016) Targeting of Frr/Cdh1 for timely activation of the APC/C at the centrosome during mitotic exit. Nat. Commun. 7, 12607

12. Pfleger, C.M. et al. (2001) Inhibition of Cdh1-APC by the MAD2-related protein MAD2L2: a novel mechanism for regulating Cdh1. Genes Dev. 15, 1759-1764

13. Barford, D. (2011) Structural insights into anaphase-promoting complex function and mechanism. Philos. Trans. R. Soc. Lond. B Biol. Sci. 366, 3605-3624

14. Thornton, B.R. et al. (2006) An architectural map of the anaphase promoting complex. Genes Dev. 20, 449-460

15. Peters, J.M. (2006) The anaphase promoting complex/cyclosome: a machine designed to destroy. Nat. Rev. Mol. Cell Biol. 7, 644-656

16. Hyun, S.Y. et al. (2013) APC/CCdh1-dependent degradation of Cdc20 requires a phosphorylation on CRY-box by pololike kinase-1 during somatic cell cycle. Biochem. Biophys. Res. Commun. 436, 12-18

17. Ohtoshi, A. et al. (2000) Human p55CDC/Cdc20 associates with cyclin A and is phosphorylated by the cyclin A-Cdk2 complex. Biochem. Biophys. Res. Commun. 268, 530-534

18. Burton, J.L. and Solomon, M.J. (2001) D box and KEN box motifs in budding yeast Hsl1p are required for APC-mediated degradation and direct binding to Cdc20p and Cdh1p. Genes Dev. 15, 2381-2395

19. Hilioti, Z. et al. (2001) The anaphase inhibitor Pds1 binds to the APC/C-associated protein Cdc20 in a destruction boxdependent manner. Curr. Biol. 11, 1347-1352 erratum 1643

20. Schwab, M. et al. (2001) Yeast Hct1 recognizes the mitotic cyclin $\mathrm{Clb2}$ and other substrates of the ubiquitin ligase APC. EMBO J. 20, 5165-5175

21. Tian, W. et al. (2012) Structural analysis of human Cdc20 supports multisite degron recognition by APC/C. Proc. Natl Acad. Sci. U.S. A. 109, 18419-18424

22. Li, D. and Roberts, R. (2001) WD-repeat proteins: structure characteristics, biological function, and their involvement in human diseases. Cell. Mol. Life Sci. 58, 2085-2097

23. Kraft, C. et al. (2005) The WD40 propeller domain of Cdh1 functions as a destruction box receptor for APC/C substrates. Mol. Cell 18, 543-553

24. Vodermaier, H.C. et al. (2003) TPR subunits of the anaphase promoting complex mediate binding to the activator protein CDH1. Curr. Biol. 13, 1459-1468

25. Izawa, D. and Pines, J. (2011) How APC/C-Cdc20 changes its substrate specificity in mitosis. Nat. Cell Biol. 13, 223-233 
26. Matyskiela, M.E. and Morgan, D.O. (2009) Analysis of activator binding sites on the APC/C supports a cooperative substrate binding mechanism. Mol. Cell 34, 68-80

27. Carroll, C.W. and Morgan, D.O. (2005) Enzymology of the anaphase-promoting complex. Methods Enzymol. 398, 219230

28. Passmore, L.A. and Barford, D. (2005) Coactivator functions in a stoichiometric complex with anaphase-promoting complex/cyclosome to mediate substrate recognition. EMBO Rep. 6, 873-878

29. Zhang, S. et al. (2016) Molecular mechanism of APC/C activation by mitotic phosphorylation. Nature 533, 260-264

30. Ge, S. et al. (2009) APC/C- and Mad2-mediated degradation of Cdc20 during spindle checkpoint activation. Cell Cycle 8, $167-171$

31. Tang, Z. et al. (2001) MAD2-independent inhibition of APCCdc20 by the mitotic checkpoint protein BubR1. Dev. Cell 1, 227-237

32. Jia, L. et al. (2016) The Bub1-Plk1 kinase complex promotes spindle checkpoint signalling through Cdc20 phosphorylation. Nat. Commun. 7, 10818

33. Lischetti, T. et al. (2014) The internal Cdc20 binding site in BubR1 facilitates both spindle assembly checkpoint signaling and silencing. Nat. Commun. 5, 5563

34. Di Fiore, B. et al. (2015) The ABBA motif binds APC/C activators and is shared by APC/C substrates and regulators. Dev. Cell 32, 358-372

35. Diaz-Martinez, L.A. et al. (2015) The Cdc20-binding Phe box of the spindle checkpoint protein BubR1 maintains the mitotic checkpoint complex during mitosis. J. Biol. Chem. 290, 2431-2443

36. Hara, M. et al. (2015) Structure of an intermediate conformer of the spindle checkpoint protein Mad2. Proc. Natl Acad. Sci. U.S.A. $112,11252-11257$

37. Luo, X. et al. (2000) Structure of the Mad2 spindle assembly checkpoint protein and its interaction with Cdc20. Nat. Struct. Biol. 7, 224-229

38. Luo, X. et al. (2002) The Mad2 spindle checkpoint protein undergoes similar major conformational changes upon binding to either Mad1 or Cdc20. Mol. Cell 9, 59-71

39. Sironi, L. et al. (2002) Crystal structure of the tetrameric Mad1-Mad2 core complex: implications of a 'safety belt' binding mechanism for the spindle checkpoint. EMBO J. 21, 2496-2506

40. Kulukian, A. et al. (2009) Unattached kinetochores catalyze production of an anaphase inhibitor that requires a Mad2 template to prime Cdc20 for BubR1 binding. Dev. Cell 16, 105-117

41. Chao, W.C.H. et al. (2012) Structure of the mitotic checkpoint complex. Nature 484, 208-213

42. Fujimitsu, K. et al. (2016) Cyclin dependent kinase 1-dependent activation of APC/C ubiquitin ligase. Science 352, 11211124

43. Qiao, R. et al. (2016) Mechanism of APC/CCDC20 activation by mitotic phosphorylation. Proc. Natl Acad. Sci. U.S.A. 113, E2570-E2578

44. Kramer, E.R. et al. (2000) Mitotic regulation of the APC activator proteins CDC20 and CDH1. Mol. Biol. Cell 11, 15551569

45. Steen, J.A. et al. (2008) Different phosphorylation states of the anaphase promoting complex in response to antimitotic drugs: a quantitative proteomic analysis. Proc. Natl Acad. Sci. U.S.A. 105, 6069-6074 
46. Lu, D. et al. (2014) Multiple mechanisms determine the order of APC/C substrate degradation in mitosis. J. Cell Biol. 207, 23-39

47. Izawa, D. and Pines, J. (2015) The mitotic checkpoint complex binds a second CDC20 to inhibit active APC/C. Nature $517,631-634$

48. London, N. and Biggins, S. (2014) Signalling dynamics in the spindle checkpoint response. Nat. Rev. Mol. Cell Biol. 15, 736-747

49. Yamaguchi, M. et al. (2016) Cryo-EM of mitotic checkpoint complex-bound APC/C reveals reciprocal and conformational regulation of ubiquitin ligation. Mol. Cell 63, 593-607

50. Alfieri, C. et al. (2016) Molecular basis of APC/C regulation by the spindle assembly checkpoint. Nature 536, 431-436

51. Vanoosthuyse, V. et al. (2009) Bub3p facilitates spindle checkpoint silencing in fission yeast. Mol. Biol. Cell 20, 50965105

52. Windecker, H. et al. (2009) Bub1 and Bub3 promote the conversion from monopolar to bipolar chromosome attachment independently of shugoshin. EMBO Rep. 10, 1022-1028

53. Craney, A. et al. (2016) Control of APC/C-dependent ubiquitin chain elongation by reversible phosphorylation. Proc. Natl Acad. Sci. U.S.A. 113, 1540-1545

54. Grallert, A. et al. (2015) A PP1-PP2A phosphatase relay controls mitotic progression. Nature 517, 94-98

55. Moura, I.M. et al. (2014) High CDC20 expression is associated with poor prognosis in oral squamous cell carcinoma. J. Oral Pathol. Med. 43, 225-231

56. Choi, J-W. et al. (2013) High expression of spindle assembly checkpoint proteins CDC20 and MAD2 is associated with poor prognosis in urothelial bladder cancer. Virchows Arch. 463, 681-687

57. Chang, D.Z. et al. (2012) Increased CDC20 expression is associated with pancreatic ductal adenocarcinoma differentiation and progression. J. Hematol. Oncol. 5, 15

58. Wu, W. et al. (2013) CDC20 overexpression predicts a poor prognosis for patients with colorectal cancer. J. Transl. Med.

11,142

59. Karra, H. et al. (2014) Cdc20 and securin overexpression predict short-term breast cancer survival. Br. J. Cancer 110, 2905-2913

60. Mao, D.D. et al. (2015) A CDC20-APC/SOX2 signaling axis regulates human glioblastoma stem-like cells. Cell Rep. 11, 1809-1821

61. Kato, T. et al. (2012) Overexpression of CDC20 predicts poor prognosis in primary non-small cell lung cancer patients. J. Surg. Oncol. 106, 423-430

62. Li, J. et al. (2014) Increased CDC20 expression is associated with development and progression of hepatocellular carcinoma. Int. J. Oncol. 45, 1547-1555

63. Taniguchi, K. et al. (2008) Targeting of CDC20 via small interfering RNA causes enhancement of the cytotoxicity of chemoradiation. Anticancer Res. 28, 1559-1563

64. Min, M. et al. (2012) How viruses affect the cell cycle through manipulation of the APC/C. Trends Microbiol. 20, 440448

65. Kapanidou, M. and Bolanos-Garcia, V.M. (2014) Spindle assembly checkpoint (SAC): more new targets for anti-cancer drug therapies. Adv. Cancer Drug Targets 54-79 
66. Miao, S. et al. (2014) Synuclein g compromises spindle assembly checkpoint and renders resistance to antimicrotubule drugs. Mol. Cancer Ther. 13, 699-713

67. Laufer, R. et al. (2014) Discovery of inhibitors of the mitotic kinase TTK based on N-(3-(3-sulfamoylphenyl)-1H-indazol-5yl)-acetamides and carboxamides. Bioorg. Med. Chem. 22, 4968-4997

68. Tsai, C.J. and Nussinov, R. (2013) The molecular basis of targeting protein kinases in cancer therapeutics. Semin. Cancer Biol. 23, 235-242

69. Montero, A. et al. (2005) Docetaxel for treatment of solid tumours: a systematic review of clinical data. Lancet Oncol. 6, 229-239

70. Brito, D.A. and Rieder, C.L. (2009) The ability to survive mitosis in the presence of microtubule poisons differs significantly between human nontransformed (RPE-1) and cancer (U2OS, HeLa) cells. Cell Motil. Cytoskeleton 66, 437-447

71. Gascoigne, K.E. and Taylor, S.S. (2009) How do anti-mitotic drugs kill cancer cells? J. Cell Sci. 122, 2579-2585

72. Frey, A. (2013) Cdc20 turnover rate: a key determinant in cancer patient response to anti-mitotic therapies? Bioessays 35,762

73. Zhou, Z. et al. (2016) Insights into APC/C: from cellular function to diseases and therapeutics. Cell Div. 11, 9

74. Sackton, K.L. et al. (2014) Synergistic blockade of mitotic exit by two chemical inhibitors of the APC/C. Nature 514, 646649

75. Stanger, K.J. et al. (2006) Synthesis and screening of $\mathrm{N}$-alkyl hydroxamates for inhibition of cancer cell proliferation. Comb. Chem. High Throughput Screen. 9, 651-661

76. Jiang, J. et al. (2012) NAHA, a novel hydroxamic acid-derivative, inhibits growth and angiogenesis of breast cancer in vitro and in vivo. PLoS One 7, e34283

77. Jiang, J. et al. (2011) Ganodermanontriol (GDNT) exerts its effect on growth and invasiveness of breast cancer cells through the down-regulation of CDC20 and uPA. Biochem. Biophys. Res. Commun. 415, 325-329

78. Lub, S. et al. (2016) Inhibiting the anaphase promoting complex/cyclosome induces a metaphase arrest and cell death in multiple myeloma cells. Oncotarget 7, 4062-4076

79. Jiang, J. and Sliva, D. (2010) Novel medicinal mushroom blend suppresses growth and invasiveness of human breast cancer cells. Int. J. Oncol. 37, 1529-1536

80. Regenbrecht, C.R. et al. (2008) The molecular basis of genistein induced mitotic arrest and exit of self-renewal in embryonal carcinoma and primary cancer cell lines. BMC Med. Genomics 1, 49

81. Zhang, L. et al. (2015) Potential therapeutic mechanism of genistein in breast cancer involves inhibition of cell cycle regulation. Mol. Med. Rep. 11, 1820-1826

82. Jamal, S. et al. (2014) CARP-1 functional mimetics are a novel class of small molecule inhibitors of malignant pleural mesothelioma cells. PLoS One 9, e89146

83. Puliyappadamba, V.T. et al. (2011) Antagonists of anaphase-promoting complex (APC)-2-cell cycle and apoptosis regulatory protein (CARP)-1 interaction are novel regulators of cell growth and apoptosis. J. Biol. Chem. 286, 38000-38017

84. Nasr, T. et al. (2014) Anticancer activity of new coumarin substituted hydrazide-hydrazone derivatives. Eur. J. Med. Chem. 76, 539-548

85. Majumder, P. et al. (2014) Inhibiting tumor growth by targeting liposomally encapsulated CDC20 siRNA to tumor vasculature: therapeutic RNA interference. J. Control. Release 180, 100-108

86. Xie, Q. et al. (2015) CDC20 maintains tumor initiating cells. Oncotarget 6, 13241-13254 
87. Brown, N.G. et al. (2016) Dual RING E3 architectures regulate multiubiquitination and ubiquitin chain elongation by APC/C. Cell 165, 1440-1453

88. Chang, L. et al. (2015) Atomic structure of the APC/C and its mechanism of protein ubiquitination. Nature $522,450-454$

89. Kidokoro, T. et al. (2008) CDC20, a potential cancer therapeutic target, is negatively regulated by p53. Oncogene 27, 1562-1571

90. Wang, Z. et al. (2013) Cdc20: a potential novel therapeutic target for cancer treatment Curr. Pharm. Des. 19, 32103214

91. Bai, X.C. et al. (2015) How cryo-EM is revolutionizing structural biology. Trends Biochem. Sci. 40, 49-57

92. Hu, K. et al. (2014) Targeting the anaphase-promoting complex/cyclosome (APC/C)-bromodomain containing 7 (BRD7) pathway for human osteosarcoma. Oncotarget 5, 3088-4100

93. Zeng, X. et al. (2010) Pharmacologic inhibition of the anaphase promoting complex induces a spindle checkpointdependent mitotic arrest in the absence of spindle damage. Cancer Cell 18, 382-395

94. Das, T. et al. (2014) Withaferin A modulates the spindle assembly checkpoint by degradation of Mad2-Cdc20 complex in colorectal cancer cell lines. Biochem. Pharmacol. 91, 31-39 
Trends

Phosphorylation and dephosphorylation cascades acting on cell-division cycle protein 20 homologue (Cdc20) control cell division and genome stability.

Various pools of $\mathrm{Cdc} 20$ complexes exist in the cell and undergo dynamic remodelling.

Cdc20 is abnormally expressed in a wide range of tumours.

Cdc20 constitutes a potential novel target for the treatment of cancer.

The combination of traditional structural biology approaches with emerging technologies such as single-particle methods and high-resolution cryo-electron microscopy has enabled us to learn new details of Cdc20 functions in health and disease. These technological advances have facilitated a mechanistic understanding of $\mathrm{Cdc} 20$-dependent allosteric regulation of the anaphase-promoting complex or cyclosome (APC/C) at an unprecedented level of detail. 
Aneuploidy: a prevalent form of genetic instability observed in many types of human cancer. Aneuploidy is a condition in which premature separation of sister chromatids results in the loss or gain of chromosomes in daughter cells.

Chemical space: a key concept in drug discovery that refers to the ensemble of all possible chemical compounds adhering to a given set of construction principles and boundary conditions to be considered in the search for new drugs.

Degron: a specific protein degradation signal that is required for the regulated destruction of the protein degradation rate. A degron can be a short amino acid sequence or a structural motif. Some central components of the SAC, including Cdc20, contain multiple degrons.

Hop1p, Rev7p, and MAD2 (HORMA) domain: functions as an adaptor for the recruitment of other proteins. Proteins containing this domain are involved in SAC and meiotic checkpoint signalling and in the regulation of meiotic recombination.

Kinetochore: from the Greek kinētikos, 'of motion', and chōros, 'place' refers to the site for attachment of chromosomes to the microtubule spindle fibers that pull sister chromatids apart during mitosis and meiosis.

Single-particle analysis: a computerised image-processing technique where projection images of individual macromolecular complexes (a.k.a. particles) from cryo-EM samples are sorted to build a 3D reconstruction of the complexes, often with near-atomic resolution.

Spindle assembly checkpoint (SAC): an evolutionarily conserved safety device of eukaryotic organisms that ensures the fidelity of chromosome segregation on cell division. During mitosis or meiosis, the SAC prevents anaphase onset until all chromosomes are properly attached to spindle microtubules.

TNM staging system: the most widely used system to describe the stage of a cancer originating from a solid tumour. Most hospitals and medical centres use the TNM system as their main method for cancer reporting. ' $T$ ' refers to the size and extent of the main (usually referred to as the primary) tumour, ' $N$ ' to the number of nearby lymph nodes that have cancer, and ' $\mathrm{M}$ ' to whether the cancer has metastasised (i.e., spread from the primary tumour to other parts of the body). 
A

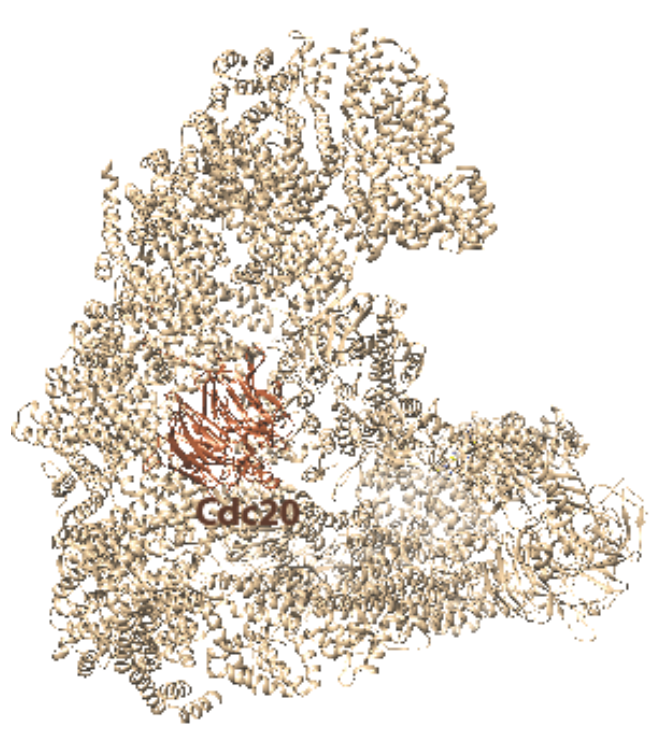

B

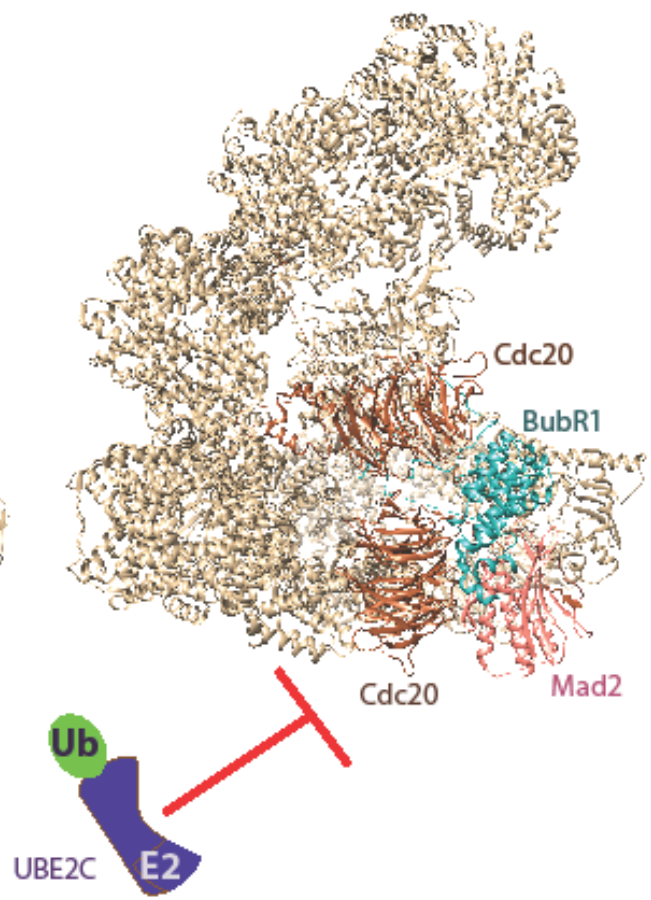

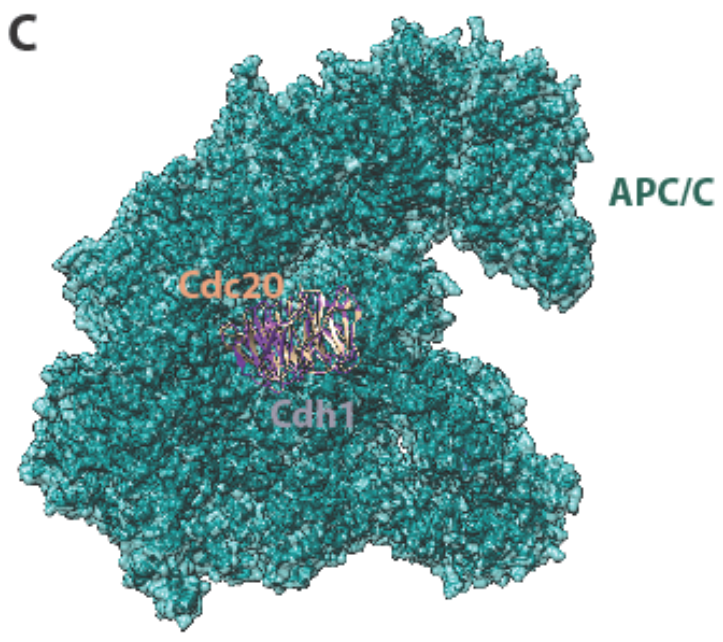

Box 1. Figure I. A Simplified View of Anaphase-Promoting Complex or Cyclosome (APC/C) Regulation by Cell-Division Cycle Protein 20 Homologue (Cdc20). (A) Cdc20 binds to APC/C and recruits APC/C substrates through interactions with short, linear amino acid sequences (degrons) present in the target proteins. (B) When forming part of the mitotic checkpoint complex (MCC), Cdc20 binds to APC/C-Cdc20 to form an APC/C-Cdc20-MCC assembly that adopts two main conformational states: open and closed. In the open conformational state, the APC/C-Cdc20-MCC assembly is not in close proximity to $A P C / C^{\prime}$ s catalytic core and the E2 coenzyme, which normally provides ubiquitin for transfer to target proteins. In the closed conformational state, $\mathrm{Cdc} 20$ bound to the MCC and budding uninhibited by benzimidazoles-related 1 (BubR1) define a continuous surface that interacts with Cdc20-APC/C $[49,50]$ thus regulating allosterically the UBE2C-dependent ubiquitination of $A P C / C$ substrates. (C) Structure superposition of the APC/C-Cdh1 and APC/C-Cdc20 complexes reveals similar modes of binding of the two WD40 proteins to APC/C. 


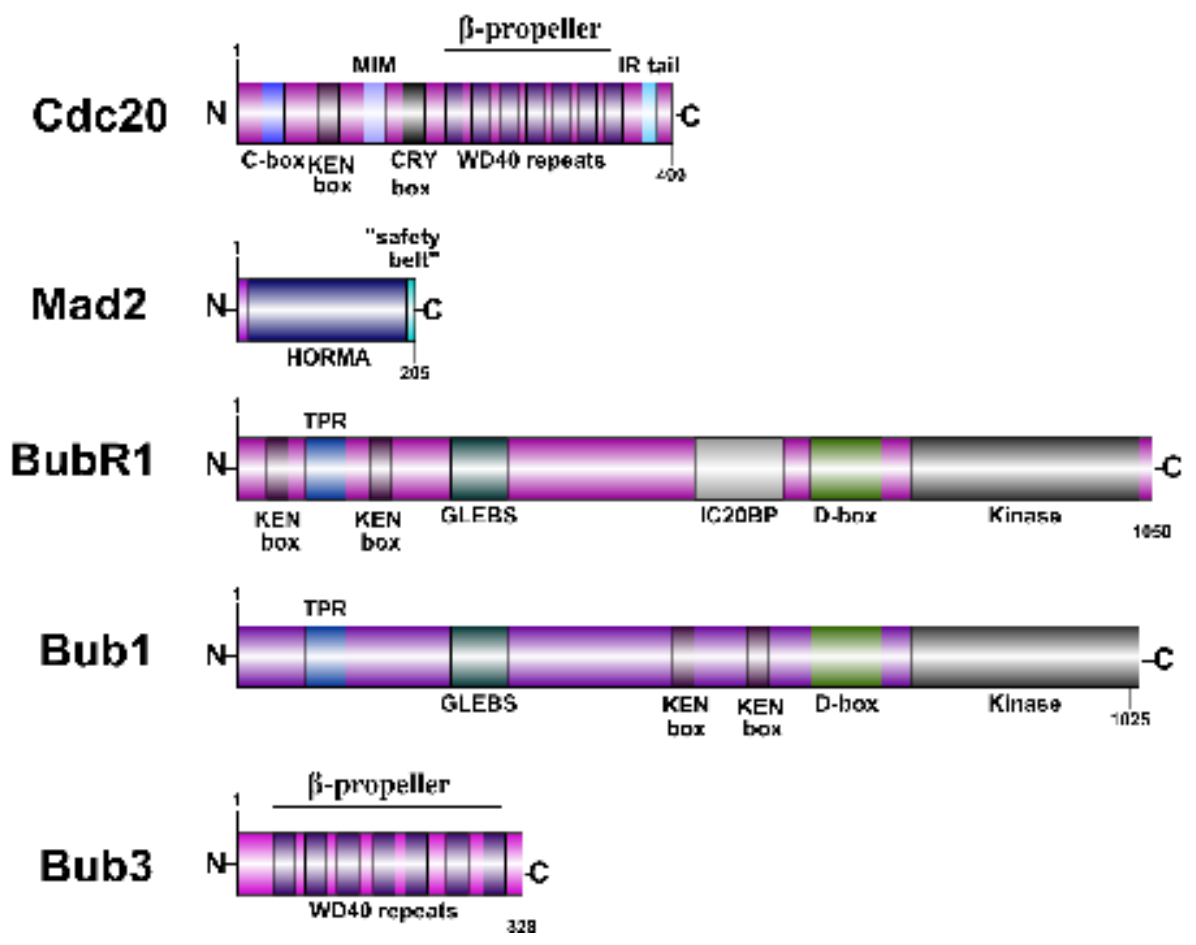

Figure 1. Domain Organisation of Cell-Division Cycle Protein 20 Homologue (Cdc20) and Core Spindle Assembly Checkpoint (SAC) Proteins. Cdc20 contains diverse motifs that mediate its multiple functions in the SAC including its binding to mitotic arrest deficient-like 2 (Mad2) and budding uninhibited by benzimidazoles (Bub)-related 1 (BubR1) and its phosphorylation by Bub1. Bub1 and BubR1 also contain diverse degrons. Mad2 is mainly organised in a Hop1p, Rev7p, and MAD2 (HORMA) domain. Bub3 shares a similar fold (WD40 repeat) with Cdc20. 
A

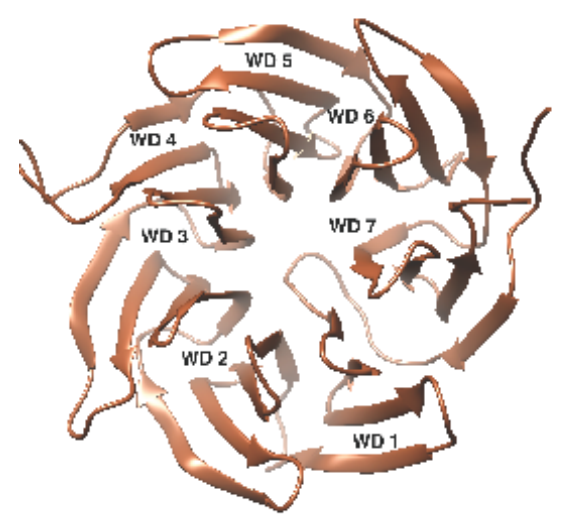

B

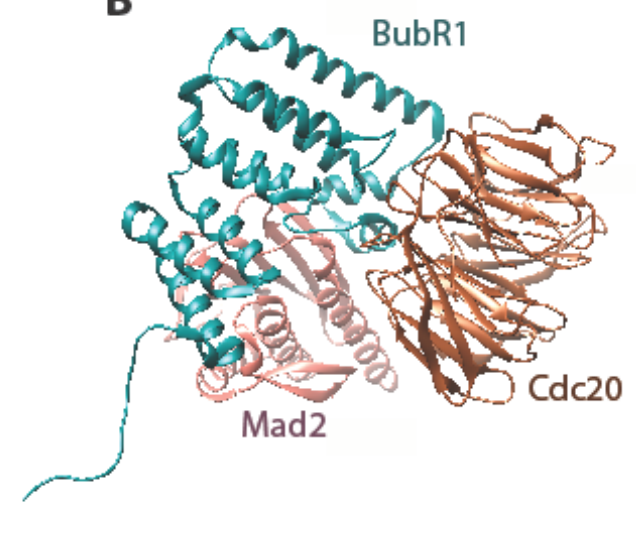

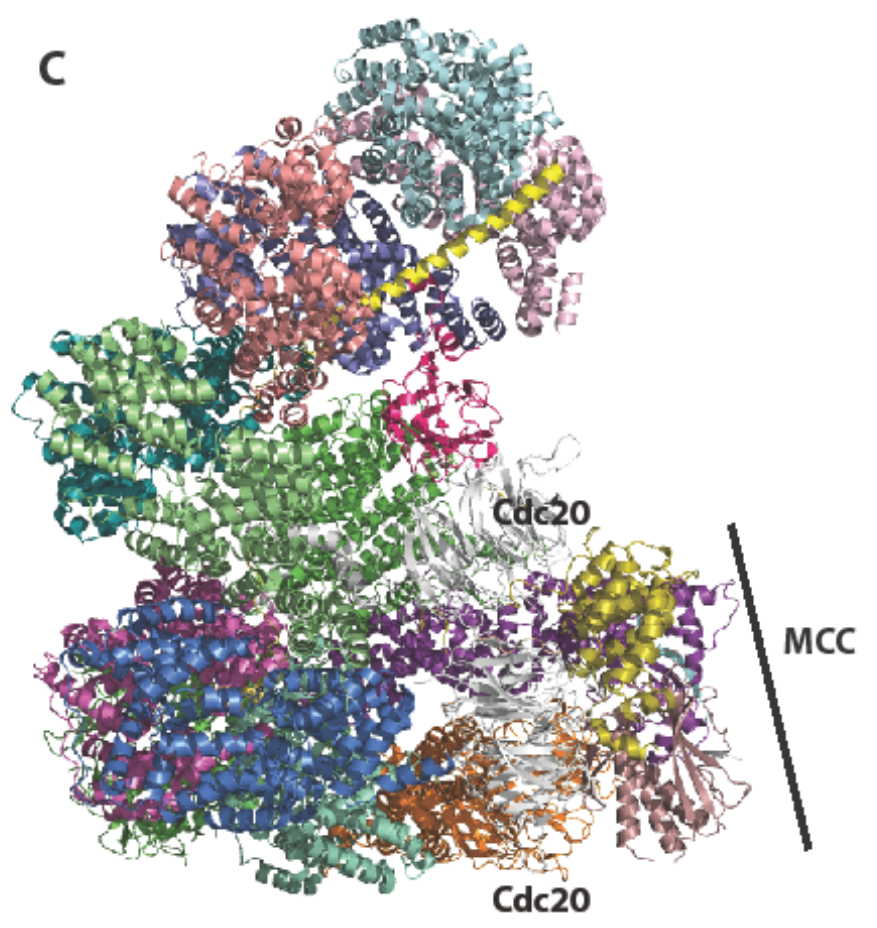

Figure 2. Cell-Division Cycle Protein 20 Homologue (Cdc20) Forms Diverse Complexes. (A) Crystal structure of a Cdc20 fragment lacking the $\mathrm{N}$-terminal region revealed a WD40-repeat domain comprising a seven-bladed b-propeller topology (PDB 4GGD). (B) 3D crystal structure of the mitotic checkpoint complex (MCC) from fission yeast (PDB 4AEZ) comprising the proteins $\mathrm{Cdc20}$, mitotic arrest deficient-like 2 (Mad2), and the $\mathrm{N}$-terminal budding uninhibited by benzimidazoles- related 1 (BubR1) (Mad3 in yeast). (C) Cryoelectron microscopy (cryo-EM) structure model of anaphase promoting complex or cyclosome (APC/C)-Cdc20-MCC. Because phase information is preserved in cryo-EM images as opposed to $X$-ray crystallographic reflections, cryo-EM maps can provide more structural details of large and rigid complexes than $\mathrm{X}$-ray electron-density maps at the same nominal resolution [91]. 


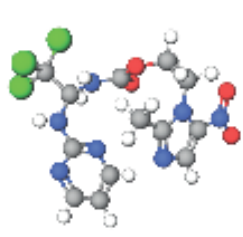

Apcin
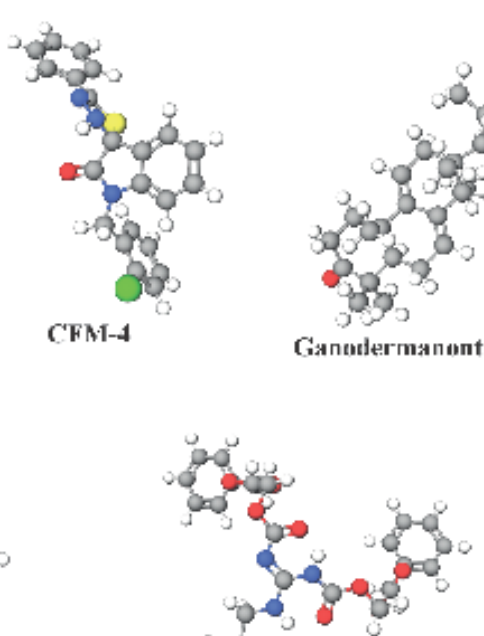
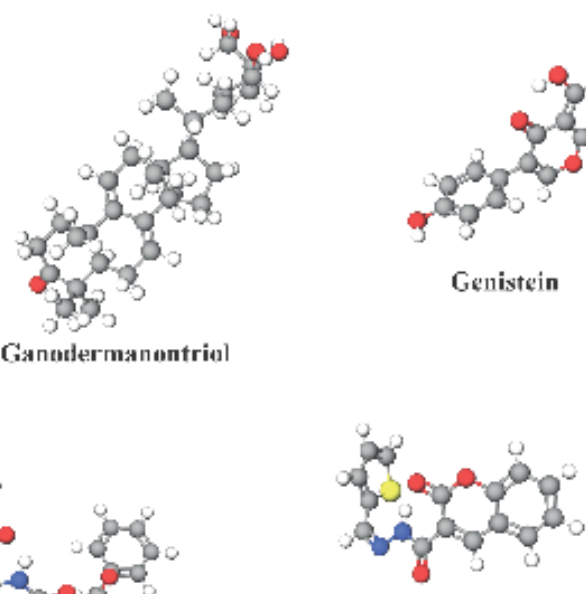

BCHHD $7 \mathrm{c}$

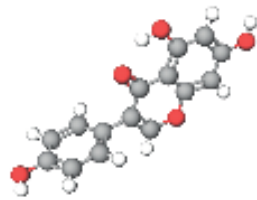

Genistein
TAMIE,

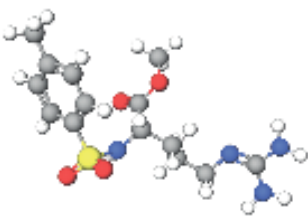

TAM

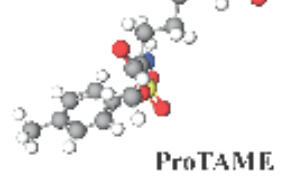

ProTAME

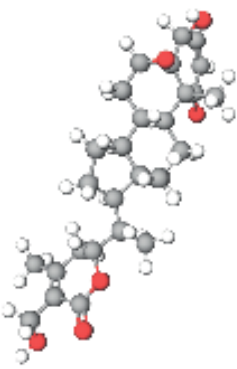

Withalerín A

Figure 3. Cell-Division Cycle Protein 20 Homologue (Cdc20) Inhibitors. Chemical structures of the small-sized inhibitors of Cdc20 reported to date. The compounds belong to different chemical families suggesting that an ample chemical space can be exploited for the design of new inhibitors of higher potency and/or specificity. 
Table 1. Small-Sized Inhibitors of APC/C-Cdc20 Functions

\begin{tabular}{|c|c|c|}
\hline $\begin{array}{l}\text { Compound } \\
\text { name }\end{array}$ & $\begin{array}{l}\text { Mode } \\
\text { of action }\end{array}$ & Reference \\
\hline Apcin & $\begin{array}{l}\text { Competes with the D-box-binding pocket thus obstructing } \\
\text { APC/C substrate loading. }\end{array}$ & {$[66]$} \\
\hline $\mathrm{BCHHD} \mathrm{7c}$ & $\begin{array}{l}\text { Suppressor of Cdc20 expression in pancreatic cancer cells } \\
\text { resistant to chemiotherapy. }\end{array}$ & [76] \\
\hline CFM-4 & $\begin{array}{l}\text { Down-regulation of Cdc20 expression in breast cancer } \\
\text { cells. }\end{array}$ & {$[75]$} \\
\hline Genistein & Down-regulation of $\mathrm{Cdc} 20$ in diverse human tumours. & {$[72,73]$} \\
\hline $\begin{array}{l}\text { Ganodermanontriol } \\
\text { and Mycophyto } \\
\text { complex }\end{array}$ & $\begin{array}{l}\text { Down-regulation of Cdc20 expression; inhibition of cell } \\
\text { proliferation and invasion in breast tumours. }\end{array}$ & {$[69,71]$} \\
\hline NAHA & $\begin{array}{l}\text { Down-regulation of Cdc20 in breast cancer cells, } \\
\text { negatively affecting cell proliferation and colony } \\
\text { formation. }\end{array}$ & [68] \\
\hline Pro-TAME & $\begin{array}{c}\text { Disruption of the APC/C-Cdc20 interaction leading to } \\
\text { lower APC/C activity upon substrates. }\end{array}$ & {$[81]$} \\
\hline TAME & $\begin{array}{l}\text { Impairing of } \mathrm{Cdc} 20 \text { recruitment to the } \mathrm{APC} / \mathrm{C} \text { thus } \\
\text { inhibiting } \mathrm{APC} / \mathrm{C} \text { activity. }\end{array}$ & {$[82]$} \\
\hline $\begin{array}{l}\text { Taxol and } \\
\text { Nocodazole }\end{array}$ & Affects Mad2-dependant suppression of APC/C-Cdc20 & \\
\hline Withaferin A & $\begin{array}{l}\text { Enhanced Cdc20 degradation, causing } \\
\text { mitotic delay. }\end{array}$ & {$[83]$} \\
\hline
\end{tabular}


Outstanding Questions

How do Cdc20 complexes link the SAC to other signalling networks in a cell and what are the physiological implications of such molecular interactions?

What is the role of Cdc20 in the assembly/disassembly of the APC/C in time and space?

How do phosphorylation and dephosphorylation cascades acting on Cdc20 affect cell division and genome stability?

How exactly do the various subcellular pools of Cdc20 ensure proper chromosome segregation?

Can the APC/C subunits play different roles independent of the APC/C holoenzyme? 